\title{
Prostate cancer brachytherapy: guidelines overview
}

\author{
Piotr Wojcieszek, MD, Brygida Białas, MD, PhD \\ Brachytherapy Department, Maria Sklodowska-Curie Memorial Cancer Centre and Institute of Oncology, Gliwice, Poland
}

\begin{abstract}
Prostate cancer, due to wide availability of PSA tests, is very often diagnosed in early stage, nowadays. This makes management of this disease even harder in every day oncology care. There is a wide range of treatment options including surgery, radiotherapy and active surveillance, but essential question is which treatment patient and oncologist should decide for. Due to recent publication of Prostate Cancer Results Study Group, in which brachytherapy is one of supreme curative options for prostate cancer, we decided to overview most present european and north american recommendations. National Comprehensive Cancer Network, American Society for Radiation Oncology, American Brachytherapy Society, European Association of Urology and Groupe Européen de Curiethérapie of European Society for Therapeutic Radiation Oncology guidelines are overviewed, particularly focusing on HDR and LDR
\end{abstract} brachytherapy.

J Contemp Brachyther 2012; 4, 2: 116-120 DOI: $10.5114 / \mathrm{jcb} .2012 .29370$

Key words: temporary implant, permanent seeds, dose prescription, dose constrains, follow-up.

\section{Purpose}

Prostate cancer becomes one of the most common cancers in developed and developing countries [1-3]. It is because life expectancy rised; age is one of the prostate cancer risk factors. Moreover there is wide availability of prostate-specific antigen (PSA) serum test, which makes possible to diagnose clinically silent, low stage disease [4]. Number of such patients is growing and makes management of this disease even harder in every day oncology care; optimal management is still controversial. There is a wide range of treatment methods and one of the most important outcome risk factors is local tumor control. There are risk categories, in which are propositions of treatment (Table 1). Very low risk category was introduced by National Comprehensive Cancer Center (NCCN) in 2010 [5].

Radical prostatectomy (RP) remains one of basic curative therapy for prostate cancer, especially for low and inermediate risk patients. PSA after radical surgery should decrease below $0,2 \mathrm{ng} / \mathrm{ml}$ and remain stable. Complications include perioperative short- term side effects as blood loss, wound infection or from anesthesia, and long-term, postoperative, as incontinence or impotence [5-8].

Radiotherapy is a good alternative for prostate cancer patients. Improved local control can be achieved with dose escalation protocols [9]. There are several tools for high dose radiotherapy. Three-dimensional conformal radiotherapy (3DCRT), dynamic techniques like intense-modulated (IMRT) or arc radiotherapy and extracranial stereotactic techniques allow to prepare normal tissue sparing plans with clinical target volume (CTV) high dose prescription. Not only photon beams, but also particles, e.g. protons are used for highly conformal irradiation [10]. Every day image-guided radiotherapy (IGRT) use, especially with intraprostatic seed markers, makes possible dose escalation and less toxicity with smaller margins contouring [11]. Brachytherapy, combined with external-beam radiotherapy (EBRT) or alone, remains one of best tools for absolute dose escalation inside prostate. Due to low alpha/beta ratio for prostate cancer these protocols are favorable, in which hypofractionation is used. Both low-dose (LDR) and high-dose rate (HDR) brachytherapy (BT) are used in prostate cancer patients. Iodine-121 ( ${ }^{121} \mathrm{I}$; mean photon energy - $27 \mathrm{keV}$, halflife: 59 days) or palladium-103 $\left({ }^{103} \mathrm{Pd}\right.$; mean photon energy $-21 \mathrm{keV}$, half-life: 17 days) are used mostly to prepare permanent LDR implant. HDR brachytherapy is, particularly, based on iridium-192 (192Ir; mean photon energy $400 \mathrm{keV}$, half-life: 74 days) stepping source, which is driven into temporary implant catheters by computer-controlled unit. Low mean photon energy is a short range of irradiation. It means that volume of irradiated tissue is relatively low comparing to EBRT. It also makes high dose escalation possible, but interstitial implant is needed [12,13]. Radiotherapy complications are acute and late, mostly in bladder and rectum, like incontinence, diarrhea, urinary stricture, impotence and proctitis. Outcome is similar to surgery, however tolerance of treatment seems better for radiotherapy excluding proctitis rates, which are higher in irradiated patients. Patient for RT are usually those, who cannot undergo surgery, older than 65 years or with locally advanced disease, e.g. extracapsular spread [5,14,15].

Active surveillance is also available as a treatment recommendation. Patients with shorter life expectancy or inconvinced to treat their disease are recommended for PSA 
Table 1. Risk categories and treatment options according to NCCN [5]

\begin{tabular}{|c|c|c|}
\hline Risk category & Life expectancy & Treatment recommendation \\
\hline \multirow[b]{2}{*}{$\begin{array}{l}\text { Very low: } \\
\text { T1c; Gleason score } \leq 6 \\
\text { PSA }<10 \mathrm{ng} / \mathrm{mL} ; \\
<3 \text { positive biopsy cores; } \\
\leq 50 \% \text { cancer in each core; } \\
\text { PSAD* }<0.15 \mathrm{ng} / \mathrm{mL} / \mathrm{g}\end{array}$} & $<20$ years & active surveillance \\
\hline & $\geq 20$ years & $\begin{array}{l}\text { active surveillance } \\
\text { or } \\
\text { radiotherapy (3DCRT/IMRT + IGRT } T^{\dagger} \text { or } \mathrm{BT} \text { ) } \\
\text { or } \\
\mathrm{RP} \pm \text { pelvic lymph node dissection }{ }^{\ddagger}\end{array}$ \\
\hline \multirow{2}{*}{$\begin{array}{l}\text { Low: } \\
\text { T1-T2a; Gleason score } \leq 6 \\
\text { PSA }<10 \text { ng/mL }\end{array}$} & $<10$ years & active surveillance \\
\hline & $\geq 10$ years & $\begin{array}{l}\text { active surveillance } \\
\text { or } \\
\text { radiotherapy (3DCRT/IMRT + IGRT' or BT) } \\
\text { or } \\
\mathrm{RP} \pm \text { pelvic lymph node dissection }{ }^{\ddagger}\end{array}$ \\
\hline \multirow[t]{2}{*}{$\begin{array}{l}\text { Intermediate: } \\
\text { T2b-T2c; Gleason score 7; } \\
\text { PSA = } 10-20 \text { ng/mL }\end{array}$} & $<10$ years & $\begin{array}{l}\text { active surveillance } \\
\text { or } \\
\text { radiotherapy (3DCRT/IMRT + IGRT } \pm B T \text { boost) } \pm \text { short-term ADT§ (4-6 months) }\end{array}$ \\
\hline & $\geq 10$ years & $\begin{array}{l}\text { radiotherapy (3DCRT/IMRT + IGRT } \pm \text { BT boost) } \pm \text { short-term ADT§ (4-6 months) } \\
\text { or } \\
\mathrm{RP} \pm \text { pelvic lymph node dissection }{ }^{\ddagger}\end{array}$ \\
\hline $\begin{array}{l}\text { High: } \\
\text { T3a; Gleason score 8-10; } \\
\text { PSA > } 20 \text { ng/mL }\end{array}$ & $\begin{array}{l}\text { If } \leq 5 \text { years } \\
\text { and asymptomatic } \\
\text { and if complication } \\
\text { as hydronephrosis } \\
\text { or dissemination } \\
\text { are expected within } \\
5 \text { years RT or ADT } \\
\text { may be considered }\end{array}$ & $\begin{array}{l}\text { radiotherapy (3DCRT/IMRT + IGRT })+ \text { long-term ADT§ (2-3 years) } \\
\text { or } \\
\text { radiotherapy (3DCRT/IMRT + IGRT }+ \text { BT boost) } \pm \text { short-term ADT§ (4-6 months) } \\
\text { or } \\
\mathrm{RP}+\text { pelvic lymph node dissection` }\end{array}$ \\
\hline $\begin{array}{l}\text { Very high: } \\
\geq T 3 b\end{array}$ & $\begin{array}{l}\text { If } \leq 5 \text { years } \\
\text { and asymptomatic } \\
\text { and if complication } \\
\text { as hydronephrosis } \\
\text { or dissemination } \\
\text { are expected within } \\
5 \text { years RT or ADT } \\
\text { may be considered }\end{array}$ & 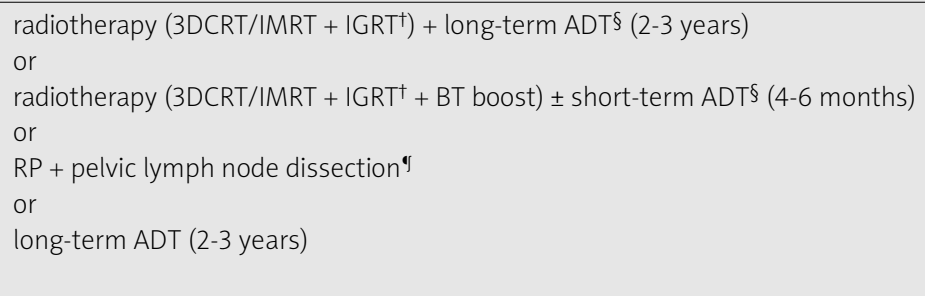 \\
\hline
\end{tabular}

testing at least every six months, digital rectal examination (DRE) at least every twelve months and, especially those with longer life expectancy, for repeated prostate biopsy at least every year. If there is disease progression observed, then patient should undergo clinical evaluation to choose optimal treatment option. Although clinical progression is still not well defined and requires physician judgement and treatment option should be chosen wisely after patient discussion with his doctor $[5,16]$.

Comparative analysis of PSA free survival outcomes by Prostate Cancer Results Study Group has been published, recently [17]. It contains comparison of results from 848 articles selected from over 18000 published between 2000 and 2010. Only radical treatment was considered containing BT (including HDR), EBRT (including IMRT), RP, proton thereapy, HIFU and cryotherapy. PCRSG agreed unanimously for study inclusion criteria as: patient stratification into pretreatment groups (according to D' Amico, Zelefsky or NCCN), PSA free time endpoint (ASTRO, Phoenix, PSA $<0,2 \mathrm{ng} / \mathrm{mL}$ for $\mathrm{RP}$ ), both clinical and pathological staging, minimum of 72 Gy EBRT (IMRT or 3DCRT), results published in peer-review journals only, minimum median follow-up of 5 years. Morover minimum patients number for each risk group was accepted as 100 for low and intermediate and 50 for high risk group. Standard deviational elipses were calculeted for each risk group. Results of this study suggest that BT alone, particularly seed implant, provides superior PSA free survival in low risk patients. EBRT combined with BT seems to be equal to BT alone, and better than EBRT alone or RP, in intermediate risk group. Combined irradiation (EBRT with BT) with or without androgen deprivation therapy seems to be superior for high-risk patients. Although these results are encouraging to choose BT as an element of managment, it should be remembered that selection bias may play main role, mostly in intermediate risk group. According to lack of large, randomized studies these may help to choose ultimate treatment decision both for physician and his patient. BT seems to be superior option for dose escalation and PSA control in prostate cancer patients. This is also reason to recall guidelines of European 
and North American societies on prostate cancer brachytherapy, both HDR and LDR.

\section{Patient selection}

European Association of Urology states that transperineal BT alone qualification criteria are: clinical stage between T1c and T2a, without nodal involvment or metastases, six or less Gleason score diagnosed with sufficient number of random biopsies, initial PSA level of $10 \mathrm{ng} / \mathrm{ml}$ or less, no more than $50 \%$ of biopsy cores with cancer, prostate volume of less than $50 \mathrm{~cm}^{3}$ and a good IPSS ( $<20$ fair tolerance; $<9$ good tolerance). LDR-BT is recommended both by EAU and GEC/ESTRO as safe and effective in low risk group of prostate cancer patients with life expectancy longer than 5 years. Post-TURP patients should avoid this procedure, however if TURP was performed several years earlier some patients can undergo LDR-BT after careful evaluation. Aspirin or anticoagulants should stop at least seven days before treatment. Any bleeding disorders are absolute contraindications $[18,19]$.

HDR-BT is recommended as a dose escalation technique combined with EBRT for patients with intermediate or high risk of failure with life expectancy longer than 5 years. The exclusion criteria are: prostate volume bigger than $60 \mathrm{~cm}^{3}$ (hormonal downsizing to reduce benign prostate hyperplasia is indicated), transurethral resection of prostate within six months, infiltration of external sphincter of the bladder neck, significant urinary obstructive symptoms (residual urine volume $>50 \mathrm{~cm}^{3}$, IPSS $>12$ and $Q_{\max }<15 \mathrm{~cm}^{3} / \mathrm{s}$ ), technical issues (pubic arch interference, TRUS prostate-rectum distance less then $5 \mathrm{~mm}$, lack of proper positioning of patient, anaesthesia or complementary EBRT contraindications). Moreover these guidelines state that HDR-BT alone should be considered as an investigational treatment with proper fractionation. Salvage HDR-BT should be also performed in a prospective clinical trial, only [20].

ABS states that HDR-BT provides highly conformal treatment with less irradiation for pelvic organs-at-risk and excellent outcome. All patients should be considered for these aproach, particularly for intermediate and high relapse risk patients as an irradiation boost or as a monotherapy for low risk patients. Salvage approach after EBRT or LDR-BT should be limited to specialty centers. Monotherapy for high risk patients should be studied in clinical trials. Special conditions are considered in ABS recommendations on HDR-BT. Prior rectal surgery or pelvic irradiation, i.e. rectal cancer treatment, in prostate cancer patients should be carefully evaluated and complications and outcome risk factors should be discussed with special HDR-BT center expertise. Inflammatory bowel disease patients should be considered for HDR-BT if not suitable for RP, asymptomatic and not required treatment for at least 0.5 to 10 years. Colonoscopy should be performed prior to treatment. HDR-BT alone seems to be better than EBRT alone or combined with HDR boost, because of smaller bowel volumes irradiated. TURP should be performed at least 90 days before HDR-BT, an aerated gel should be given into urethtra to outline its shape and special attention should be given to not exceed $110 \%$ of prescription dose. Most feasible glands for HDR-BT are those beneath $50 \mathrm{~cm}^{3}$, but ABS guidelines allow to treat larger ones. Pubic arch interference, baseline urinary function and application technique (fixed template or freehand) should be carefully considered. ADT, 5-alpha reductase or limited TURP may be chosen for cytoreduction. Patients should be informed about potential risks of erectile dysfunction or acute and late urinary side effects. ABS states that absolute contraindications include preexisting rectal fistule, impossibility of anaesthesia and no proof of malignancy [21].

LDR-BT alone is recommended by ABS for prostate cancer low risk patients. Absolute contraindications include poor performance status and life expectancy shorter than 10 years, spinal or general anaesthesia not possible, large post TURP defects and lack of rectum, which makes TRUS impossible. IPSS value over 20 points can correlate with increased urinary toxicity and needs careful evaluation. Prostate volume over $60 \mathrm{~cm}^{3}$ is not a contraindication and even with pubic arch interference can be considered for LDR-BT with short ADT course (3-4 months) to downsize the gland. Also freehand implantation is acceptable, but experienced practitioners should perform LDR-BT in such cases. ABS states that LDR-BT alone should be done in low risk patients and as option for intermediate group. EBRT and permanent seed combination is considered as standard of care for high risk patients [22].

\section{Application and treatment planning}

HDR-BT, according to GEC/ESTRO-EAU recommendations, should be done under TRUS guidance with template, its proper fixation and treatment planning software. Adequate shielding operative room is needed. Additional imaging with CT or MRI after implantation may be used. Clinical target volume (CTV) for HDR-BT can be whole gland ('prostate surface') with homogenous needles distribution or with addition of visible tumor boost inside CTV. Different approach is to put needles in the peripheral zone only. Prostate surface dose prescription differs among cancer centers and is between 6 to 10 Gy per fraction with total dose of 12-20 Gy in 2 to 4 fractions combined with EBRT of 45 to 54 Gy in 6-7 weeks. Inter-fraction time may vary from hours to two weeks gap. Organs-at-risk doses should not exceed $120 \%$ of MTD for urethra and less then 6 Gy per fraction for rectum. Those doses should be precisely stated and valuated in clinical trials with long follow-up including penile bulb maximum dose [20].

ABS consensus allows TRUS, CT or MRI for treatment planning. Proximal tips of catheters should be visualized and images should be taken at least $9 \mathrm{~mm}$ above and below CTV. Additional techniques are recommended for urethral identification. Organs-at-risk should be confined including rectum, urethra, bladder and penile bulb. Minimum of 14 catheters should be placed or more, particularly if 'boost within boost' is planned. Especially urethra piercing should be avoided. At least $90 \%$ of CTV should be covered with prescribed dose with aiming 95\% of CTV. HDR-BT boost is given in one to six and HDR-BT alone in three to six fractions. Due to wide heterogeneity of fractionation schedules, OaR constraints are based on experienced HDR centers references, e.g. from less then $105 \%$ to less then $125 \%$ for urethra [21]. 
LDR-BT should be performed under TRUS guidance according to GEC/ESTRO guidelines. If possible gross tumor volume (GTV) should be delineated on pre-treatment scans. CTV should contain whole prostate with $3 \mathrm{~mm}$ margin in all directions except posterior (rectal wall) and cranial (bladder neck) if needed. Extracapsular extension shoul be undertaken for T3 tumors. PTV is equal CTV. Prostatic urethra outline should be based on the surface of catheter. Rectal outer wall delineation is minimum. Penile bulb and neurovascular bundles remain under investigation. Dose prescription to $100 \%$ isodose should be $145 \mathrm{~Gy}$ for ${ }^{125} \mathrm{I}$ and $125 \mathrm{~Gy}$ for ${ }^{103} \mathrm{Pd}$ seeds. At least $95 \%$ of CTV should be covered with $100 \%$ isodose with no more than $50 \%$ of CTV with isodose $150 \%$. Maximum dose in $2 \mathrm{~cm}^{3}$ for rectal wall should be no more than $145 \mathrm{~Gy}$ and in $0.1 \mathrm{~cm}^{3}$ less than $200 \mathrm{~Gy} .150 \%$ of prescribed dose should cover less than $10 \%$ of urethra volume and $130 \%$ of this dose should cover less than $30 \%$ of its volume [23].

ABS recommends to use TRUS as a basic imaging modality for LDR-BT. It should be done as a preplanning, either as a separate procedure or on the implanting day in operational room. CT or MRI can also be used to prepare virtual plan. It should be done for adequate needle guidance during implantation. Main procedure should be performed with TRUS and template; patient position and probe angle should be similar to preplanned. Minimum is biplanar, high resolution (5-12 MHz) TRUS with dedicated planning software. Team should consist at least of trained radiation oncologist and qualified medical physicist, but presence of urologist, certified dosimetrist or other supportive staff is appropriate. ABS does not suggest any seed deposition technique, however it should have excellent outcome, be reproducible and with optimal dosimetry. Both stranded and loose seeds can be chosen for implantation. ${ }^{125} \mathrm{I}$ and ${ }^{103} \mathrm{Pd}$ implants showed excellent outcomes, so ABS do not favor any of them. ${ }^{131} \mathrm{Cs}$ is still under investigation in multicenter clinical trial. Previously used 198Au is not recommended for routine practice. LDR-BT alone prescription dose to PTV should range from 140-160 Gy for ${ }^{125} \mathrm{I}$ seeds and 110-125 Gy for ${ }^{103} \mathrm{Pd}$. If combination of EBRT and seed implantation is planned, ${ }^{125}$ I PTV dose should be between 108-110 Gy and ${ }^{103}$ Pd PTV dose should be between 90-100 Gy. EBRT dose should be 41.4-50.4 Gy (1.8 Gy/day, but 2 Gy/day is also acceptable). LDR-BT is usually performed 0-8 weeks after EBRT, but there is no evidence for optimal sequence of these. Reverse approach is also accepted, which may theoretically be better, because of simultaneous irradiation from implant and EBRT and possibility of adjusting EBRT if needed. It can increase toxicity, either. Dose constrains for organs at risk should not exceed $150 \%$ of prescribed dose in $5 \%$ and $125 \%$ of prescribed dose in $30 \%$ of urethral volume in the preplan. Prescribed dose should be in less then $1 \mathrm{~cm}^{3}$ of rectum on day 1 dosimetry and in less then $1.3 \mathrm{~cm}^{3}$ on day 30 . Postimplant CT-based dosimetry should be done within 60 days from the procedure. Although CT performed immediately or one day after the implantation has several positives, e.g. early detection of dosimetric problems, but after implant oedema can derive dosimetric underestimations. Optimal radionuclide-dependent CT timing is recommended. It is $12-20$ days after implantation for ${ }^{103} \mathrm{Pd}$ and $23-37$ days for ${ }^{125} \mathrm{I}$. Erectile func- tion organs at risk e.g. penile bulb are still not agreed, though there are no recommendations on these [22].

\section{Follow-up}

Both LDR and HDR-BT patients should be seen 6 weeks after implantation (last one for temporary implants) according to GEC/ESTRO recommendations. This is to evaluate acute side effects. During first year patient should be examined every 3 months, every 6 months until 5 years after treatment and then annually. DRE, PSA test, side effects evaluation (urinary, rectal, potency toxicity and IPSS) and TRUS should be done $[18,20]$.

ABS suggests to follow-up HDR-BT patients every six months for first 2-3 years, and then at least annually. It should consist of DRE, PSA test and toxicity evaluation (urinary, rectal and sexual function). Phoenix definition of biochemical relapse is recommended, although case individual assessment is required and patient should be informed about PSA bounce. LDR-BT patients should be seen every six to twelve months with regular intervals. DRE, PSA test and toxicity reports should be reported. Phoenix definition of biochemical relapse is recommended. High risk patients can be seen more frequent. After PSA rising prostate biopsy may be uninterpretable within 30 months after implantation. Post BT rectal biopsies or urinary instrumentation should be avoided and all of the risks and benefits should be considered [21,22].

\section{Summary}

Brachytherapy is supreme tool in prostate cancer management with a wide range of options in every risk group (Table 2). Excellent outcomes and relatively small irradiated volume seems more beneficial than any risk of intraoperative implantation, particularly in wisely selected patients. Feasibility of seeding or catheterization is basic for choosing appropriate candidates for BT, however there are articles describing series of patient with non-fitting anatomy, i.e. prostate smaller than $20 \mathrm{~cm}^{3}$ or bigger then $50 \mathrm{~cm}^{3}$ or post TURP patients $[24,25]$. Obesity and high body mass

Table 2. Recommendations comparison for GEC/ ESTRO and ABS [18,20-22]

\begin{tabular}{lcc} 
& $\begin{array}{c}\text { GEC/ESTRO-EAU } \\
\text { recommendations }\end{array}$ & $\begin{array}{c}\text { ABS } \\
\text { recommendations }\end{array}$ \\
\hline Monotherapy & investigational & standard* $^{*}$ \\
\hline HDR-BT & standard & standard \\
\hline LDR-BT & standard & standard \\
\hline HDR-BT & not stated & standard \\
\hline LDR-BT & investigational & limited ${ }^{\dagger}$ \\
\hline Salvage & not stated & not stated
\end{tabular}

*Under investigation for high risk patients; ${ }^{+}$to Institutional Review Board - protocols or specialty centers with appropriate expertise 
index are not contraindications for BT. It is crucial that patients are properly evaluated before choosing any treatment approach. Assesment should consist of careful clinical examination including DRE, patient's history (i.e. urologic, prior pelvic RT, surgery or trauma, inflammatory bowel disease, connective tissue dissorders), pretherapy PSA serum level, biopsy proven cancer with Gleason score and clinical staging. TRUS stays basic imaging, but CT and MRI are also very useful, either before procedure and as a planning guidance. New abilities of MRI, i.e. spectroscopy and diffusion, and 11C PET-CT should be discussed more in next recommendations [26-28]. American guidelines has been published in the beginning of 2012. GEC/ESTRO and EAU recommendations on prostate cancer treatment with temporary implants with stepping sources are from 2005 and on permanent seeds from 2000 with 2007 update. Although general approach for BT, both HDR and LDR, has not particular differences across the world, European recommendations need an update.

\section{References}

1. Siegel R, Naishadham D, Jemal A. Cancer statistics, 2012. CA Cancer J Clin 2012; 62: 10-29.

2. Gondos A, Bray F, Brewster DH et al. Recent trends in cancer survival across Europe between 2000 and 2004: a model-based period analysis from 12 cancer registries. Eur J Cancer 2008; 44 1463-1475.

3. Westlake S, Cooper N. Cancer incidence and mortality: trends in the United Kingdom and constituent countries, 1993 to 2004. Health Stat Q 2008; 38: 33-46.

4. Etzioni R, Penson DF, Legler JM et al. Overdiagnosis due to prostate-specific antigen screening: lessons from U.S. prostate cancer incidence trends. J Natl Cancer Inst 2002; 94: 981-990.

5. NCCN. Clinical Practice Guidelines in Oncology. Prostate Cancer version 3.2012, 2012

6. Kibel AS, Ciezki JP, Klein EA et al. Survival among men with clinically localized prostate cancer treated with radical prostatectomy or radiation therapy in the prostate specific antigen era. J Urol 2012; 187: 1259-1265.

7. Bill-Axelson A, Holmberg L, Filen F et al. Radical prostatectomy versus watchful waiting in localized prostate cancer: the Scandinavian prostate cancer group - 4 randomized trial. J Natl Cancer Inst 2008; 100: 1144-1154.

8. Heidenreich A, Bellmunt J, Bolla $\mathrm{M}$ et al. EAU guidelines on prostate cancer. Part 1: screening, diagnosis, and treatment of clinically localised disease. Eur Urol 2011; 59: 61-71.

9. Peeters ST, Heemsbergen WD, Koper PC et al. Dose-response in radiotherapy for localized prostate cancer: results of the Dutch multicenter randomized phase III trial comparing 68 Gy of radiotherapy with 78 Gy. J Clin Oncol 2006; 24: 1990-1996.

10. Zietman AL, Bae K, Slater JD et al. Randomized trial comparing conventional-dose with high-dose conformal radiation therapy in early-stage adenocarcinoma of the prostate: longterm results from proton radiation oncology group/american college of radiology 95-09. J Clin Oncol 2010; 28: 1106-1111.

11. Sandhu A, Sethi R, Rice R et al. Prostate bed localization with image-guided approach using on-board imaging: reporting acute toxicity and implications for radiation therapy planning following prostatectomy. Radiother Oncol 2008; 88: 20-25.

12. Haie-Meder C, Siebert FA, Potter R. Image guided, adaptive, accelerated, high dose brachytherapy as model for advanced small volume radiotherapy. Radiother Oncol 2011; 100: 333-343.

13. Rubens DJ, Yu Y, Barnes AS et al. Image-guided brachytherapy for prostate cancer. Radiol Clin North Am 2006; 44: 735-748, viii-ix.
14. Potosky AL, Legler J, Albertsen PC et al. Health outcomes after prostatectomy or radiotherapy for prostate cancer: results from the Prostate Cancer Outcomes Study. J Natl Cancer Inst 2000; 92: 1582-1592.

15. Rosenthal SA, Bittner NH, Beyer DC et al. American Society for Radiation Oncology (ASTRO) and American College of Radiology (ACR) practice guideline for the transperineal permanent brachytherapy of prostate cancer. Int J Radiat Oncol Biol Phys 2010; 79: 335-341.

16. Dall'Era MA, Cooperberg MR, Chan JM et al. Active surveillance for early-stage prostate cancer: review of the current literature. Cancer 2008; 112: 1650-1659.

17. Grimm P, Billiet I, Bostwick D et al. Comparative analysis of prostate-specific antigen free survival outcomes for patients with low, intermediate and high risk prostate cancer treatment by radical therapy. Results from the Prostate Cancer Results Study Group. BJU Int 2012; 109 Suppl 1: 22-29.

18. Ash D, Flynn A, Battermann J et al. ESTRO/EAU/EORTC recommendations on permanent seed implantation for localized prostate cancer. Radiother Oncol 2000; 57: 315-321.

19. EAU. Guidelines on Prostate Cancer (2012) 2012.

20. Kovacs G, Potter R, Loch T et al. GEC/ESTRO-EAU recommendations on temporary brachytherapy using stepping sources for localised prostate cancer. Radiother Oncol 2005; 74: 137-148.

21. Yamada Y, Rogers L, Demanes DJ et al. American Brachytherapy Society consensus guidelines for high-dose-rate prostate brachytherapy. Brachytherapy 2012; 11: 20-32.

22. Davis BJ, Horwitz EM, Lee WR et al. American Brachytherapy Society consensus guidelines for transrectal ultrasoundguided permanent prostate brachytherapy. Brachytherapy 2012; 11: 6-19.

23. Salembier C, Lavagnini P, Nickers $P$ et al. Tumour and target volumes in permanent prostate brachytherapy: a supplement to the ESTRO/EAU/EORTC recommendations on prostate brachytherapy. Radiother Oncol 2007; 83: 3-10.

24. Gibbons EP, Jacobs BL, Smith RP et al. Dosimetric outcomes in prostate brachytherapy: is downsizing the prostate with androgen deprivation necessary? Brachytherapy 2009; 8: 304-308.

25. Peddada A, Jennings S, Biggers R et al. HDR Prostate Brachytherapy in Anatomically Unsuitable Cases $(<20 \mathrm{cc},>50 \mathrm{cc}$, prior TURP) - Uncompromised Results WCB 2012/ESTRO 31. Barcelona, Spain, 2012.

26. Picchio M, Briganti A, Fanti S et al. The role of choline positron emission tomography/computed tomography in the management of patients with prostate-specific antigen progression after radical treatment of prostate cancer. Eur Urol 2011; 59: 51-60.

27. Westphalen AC, Reed GD, Vinh PP et al. Multiparametric 3T endorectal mri after external beam radiation therapy for prostate cancer. J Magn Reson Imaging 2012 Apr 25. doi: 10.1002/ jmri.23672 [Epub ahead of print].

28. Polo A. Image fusion techniques in permanent seed implantation. J Contemp Brachyther 2010; 2: 98-106. 